\begin{tabular}{|c|c|}
\hline $\begin{array}{c}\text { Mechanics Research Communications. } \\
\text { Year }\end{array}$ & Publication Office: \\
Elsevier UK \\
\hline $\begin{array}{l}\text { Editor-in-Chief: A. Rosato New Jersey Institute of Technology, Newark, New Jersey, } \\
\text { USA Anthonn:-Rosateonjit.dedu }\end{array}$ & \\
\hline
\end{tabular}

\title{
Grain- and Macro-scale Kinematics for Granular Micromechanics based Small Deformation Micromorphic Continuum Model
}

\author{
Anil Misra $^{1 *}$ and Payam Poorsolhjouy ${ }^{1}$ \\ ${ }^{I}$ Civil, Environmental and Architectural Engineering Department University of Kansas, 1530 W. 15th Street, Learned Hall, Lawrence, KS 66045-7609, USA \\ *amisra@ku.edu \\ Tel.:+1-785-864-1750; fax: +1-785-864-5631] \\ Accepted: xxxxxx.
}

\begin{abstract}
Macro-scale deformation of granular solids comprising large number of grains $\left(>10^{6}\right)$ are most efficiently described within the framework of continuum mechanics. It is notable, however that the micro-scale deformations in these materials are concentrated at the grain-boundaries or grain-contacts. Thus, the deformation energies in these systems must be modeled by considering the deformations concentrated in the neighborhood of the grain-boundaries or grain-contacts. To address this issue, grain-interactions has been widely described in the Hertzian sense by considering the relative movement of points on either side of a grain boundary or contact treated as an imperfect interface. This communication introduces the relevant kinematic variables given in the terms of the grain displacements, spins and size that can be used to estimate the relative movement of a grain boundary or contact. The macro-scale kinematic variables useful for continuum modelling are then identified with the grain-scale kinematic variables. The deformation energy density of the granular solid can thus be expressed both in terms of the grain-scale as well as the macro-scale kinematic variables providing the necessary pathway for micro-macro identification which can lead to non-classical micromorphic continuum models that incorporate grain-scale representation.
\end{abstract}

(C) 2015 The Authors. Published by Elsevier Ltd.

Keywords: granular micromechanics; micromorphic continuum; grain spins; stress-force relationships; moment stress; double stress.

\section{Introduction}

Continuum modeling continues to be attractive and, arguably, the most feasible approach for describing the mechanical response of granular solids at macro-scale. It is notable, however, that in granular materials, the micro-scale deformations are concentrated in the close neighborhood of the grain-boundaries or grain-contacts. Therefore, for granular material systems, the grain-pair interactions and granular structures have a strong effect upon the collective behavior of grains. The imperatives of including the grainscale information in the macro-scale continuum models of granular solids have been widely recognized (see among a subset of early authors [1-8] and more recent contributions [912]). Clearly, the deformation energies of these material systems must be modeled by considering the deformations concentrated in the neighborhood of the grain-boundaries or grain-contacts. To address this issue, grain-pair interactions are often treated as two solid bodies with an imperfect interface and the relative movement of the grain centroids are identified with the relative movements of points on either side of the interface, such as that exemplified by the contact theory of solid bodies [13] or mechanics of interfaces of crystals [14].

This communication focusses upon discussion of the kinematic variables necessary for determination of the grainpair relative movement and their relationships to continuum counterparts. To this end we introduce two new grain-scale kinematic measures, defined in the terms of the grain centroid displacement, spin and size; one for determining relative displacements and the other for relative rotations. These grain-scale kinematic variables are then identified with the relevant macro-scale kinematic variables useful for continuum modelling following the paradigm of granular micromechanics [15-17]. The fact that the conceptual 
material point in a continua is composed of a microstructure was recognized by the pioneers of the mathematical methods of continuum mechanics (see Navier[18], Cauchy [19], and Piola [20-22]) and variously addressed in the works of Cosserat[23], Mindlin[24], Toupin[25], Eringen[26], Green and Rivlin[27] and Germain[28]. In recent years, there is renewed attention to the generalized continuum and micromorphic models for micro-structured media [29-35] including granular media [36-39]. In addition, there is increasing interest in models that seek identification of results from discrete methods, such as molecular mechanics (MM), molecular dynamics (MD) and discrete element method (DEM), with continuum-scale kinematic and stress measures with a view of linking scales in multi-scale modeling frameworks [40-44].

The method of granular micromechanics provides a practical approach for relating micro- and macro- scales for developing continuum models of granular material systems $[17,45]$. In the case of granular solids, the microstructure is composed of distinct grains. The methodology considers grains and their interactions as the building block of the material and its macroscopic behavior. In their previous work [36], the authors extended the granular micromechanics approach by identifying the grain displacements with the continuum kinematic measures of Mindlin-Eringen microstructural elasticity [24] or micromorphic mechanics [26]. In the present work, the kinematical analysis is further enhanced by including the effects of grain spins which leads to introduction of new measures at both grain- and macro-scales. The deformation energy density of a material point can then be expressed in terms of the newly defined measures at the two-scales. The inter-granular force measures are then defined as conjugates of the grain-scale kinematic measures that include stretch/compression, tangential, bending and torsional actions. Furthermore, continuum stress measures are obtained as conjugates of the macro-scale kinematic measures. By identifying the deformation energy densities expressed in terms of the micro- and macro- kinematic measures, the relationships between stress and inter-granular forces are obtained. These relations can form the basis for further derivation of the constitutive relations, variational principle, and balance equations for a non-classical micromorphic continuum model that incorporate grain-scale representation.

\section{Kinematics of Granular Solids}

Consider the relative movement of neighboring grains $n$ and $\mathrm{p}$ in a volume element (VE) of deforming granular solid expressed as

$$
\delta_{i}^{n p}=\phi_{i}^{p}-\phi_{i}^{n} ; \quad \theta_{i}^{n p}=\kappa_{i}^{p}-\kappa_{i}^{n}
$$

where $\delta_{i}^{n p}$ denotes the relative displacement of the centroids and $\theta_{i}^{n p}$ denotes the relative rotations of grain-pair $n$ and $p$. Since the deformations are concentrated in the neighborhood of the grain-boundaries or grain-contacts, the relative movement in Eq. 1 are identified with relative movement of points on either side of a grain boundary or contact treated as an imperfect interface, a concept useful for introducing intergranular constitutive relations. Further, since grains are known to rotate in a deforming granular solid [46, 47], the displacement of a grain centroid, $\phi_{i}$, has been often decomposed as [48]

$\phi_{i}=u_{i}+e_{i j k} \kappa_{j} r_{k}$

where $u_{i}$ represents the grain centroid displacement for grains constrained from rotating or for vanishingly small grains as in atomistic models (MM and MD). And the second term in Eq. 2 represents the displacement at the interface due to grain rotations, given as the cross product the rotation, $\kappa_{j}$, with the position vector, $r_{k}$, of a point on the grain-pair interface with respect to the grains' centroids. In many previous continuum models of granular materials the variables, $u_{i}$ and $\kappa_{j}$, have been used for independent identification with continuum kinematic quantities (see $[2,48,49]$ ). Clearly the variable, $u_{i}$, represents only a part of the grain centroid displacement and alone does not describe the overall deformation of a VE of granular solid. Therefore, the total grain centroid displacement, $\phi_{i}$, is the appropriate quantity for identification with continuum kinematic variable that describes the deformation of a VE. We further note that the decomposition in Eq. 2 may be used to separately estimate the two contributions to the grain centroid displacement. However this decomposition may not be easy to estimate quantitatively since it depends upon a number of factor including grain shapes, the geometry of the interface, composition, interphase layer and roughness.

The grain rotation field can similarly be decomposed into the grain spin, $\omega_{i}$, and the curl of the grain displacement field given as

$\kappa_{i}=\frac{1}{2} e_{i j k} \phi_{k, j}+\omega_{i}$

Here again we remark that in contrast to the past representation $[48,49]$, the overall grain rotation field, $\kappa_{i}$, as opposed to the grain spin, $\omega_{i}$, best describes the relative rotation between the grains and renders redundant the need for decomposition.

For developing a continuum description, the VE plays the role of a material point $\mathrm{P}$, as depicted in Figure 1. Let us consider the continuum in the global or macro-scale coordinate system, $\boldsymbol{x}$. Further attach a local or micro-scale coordinate system, $\boldsymbol{x}^{\prime}$, to the material point or the barycenter of VE with its axes parallel to those of the global coordinate axes. The micro-scale coordinate system is defined such that it is able to distinguish different grains inside the material point. The displacements and rotations of the grains can be now written as functions of not only the coordinates of the material point $\mathrm{P}$, but also the micro-scale coordinates of the grain within the material point, i.e. $\phi_{i}\left(\boldsymbol{x}, \boldsymbol{x}^{\prime}\right)$ and $\kappa_{i}\left(\boldsymbol{x}, \boldsymbol{x}^{\prime}\right)$. For any grain, $p$, inside the VE, the vector field of displacement can be written as a Taylor series expansion in terms of the 
neighboring grain, $n$, displacements and displacement gradients as follows

$$
\phi_{i}^{p}=\phi_{i}^{n}+\phi_{i, j}^{n} l_{j}+\frac{1}{2} \phi_{i, j k}^{n} l_{j} l_{k}+\cdots=\phi_{i}^{n}+\phi_{i, j}^{n} l_{j}+\phi_{i, j k}^{n} J_{j k}+\cdots
$$

where $\phi_{i}$ is grain displacement defined in Eq. $2, l_{j}$ is an intergranular branch vector joining the centroids of grains $n$ and $p$, and the tensor product $l_{j} l_{k} / 2=J_{j k}$ is a geometry moment tensor. First and second gradient terms have been included in the analysis and summation convention over repeated indices is implied unless noted otherwise.

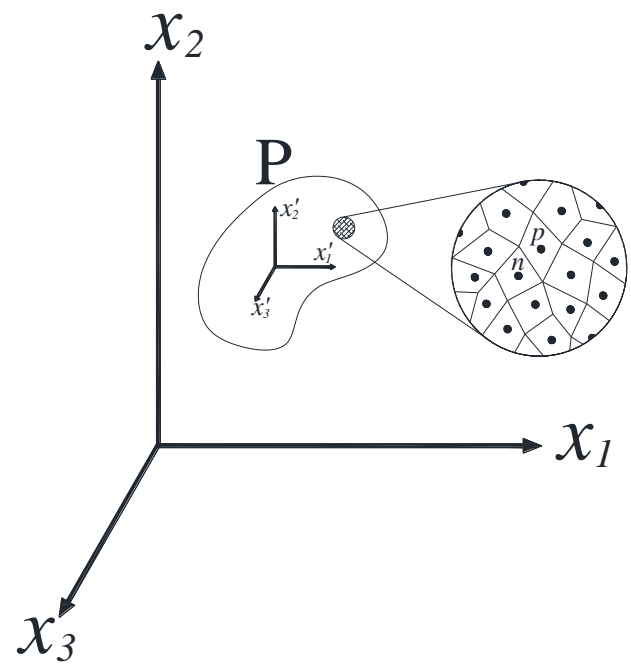

Figure 1- Schematic of continuum material point, $\mathrm{P}$, with its granular microstructure and the coordinate systems $x$ and $x$ '.

It is known that, in general, finite rotations are not vectors and do not follow vector analysis rules [50]. However, for small rotation measures, which is of interest in this study, vector analysis rules can be applied. Thus for the rotation field within the VE, the same approach as that for the displacements is used and the rotation of a grain, $p$, within the VE is written as the Taylor series expansion of the rotation of a neighbor grain, $n$.

$$
\kappa_{i}^{p}=\kappa_{i}^{n}+\kappa_{i, j}^{n} l_{j}+\frac{1}{2} \kappa_{i, j k}^{n} l_{j} l_{k}+\cdots=\kappa_{i}^{n}+\kappa_{i, j}^{n} l_{j}+\kappa_{i, j k}^{n} J_{j k}+\cdots
$$

where $\kappa_{i}$ is the rotation of the grain, $l_{j}$ is an inter-granular branch vector joining the centroids of grains $n$ and $p$, and the tensor product $l_{j} l_{k} / 2=J_{j k}$ is a geometry moment tensor.

Along the lines of the methodology of micro-structural elasticity described by Mindlin [24] and micromorphic mechanics of Eringen [26], the displacement and rotation fields within the VE are decomposed into two components, one representing an average field and the other representing the micro-scale fluctuations from average. Thus the displacement and rotation fields and their gradients can be rewritten as

$$
\phi_{i}=\bar{\phi}_{i}-\tilde{\phi}_{i} \quad \Rightarrow \quad \psi_{i j}^{\phi}=\phi_{i, j}=\bar{\phi}_{i, j}-\tilde{\phi}_{i, j}=\bar{\phi}_{i, j}-\gamma_{i j}^{\phi}
$$

$$
\kappa_{i}=\bar{\kappa}_{i}-\tilde{\kappa}_{i} \Rightarrow \psi_{i j}^{\kappa}=\kappa_{i, j}=\bar{\kappa}_{i, j}-\tilde{\kappa}_{i, j}=\bar{\kappa}_{i, j}-\gamma_{i j}^{\kappa}
$$

where the overhead bar and tilde represent the average and fluctuation fields respectively. In Eq. $6, \bar{\phi}_{i, j}$ is taken as the macro-scale gradient of the average displacement field, and $\tilde{\phi}_{i, j}=\gamma_{i j}^{\phi}$ is the micro-scale gradient of the displacement fluctuations. Similarly $\bar{\kappa}_{i, j}$ and $\tilde{\kappa}_{i, j}=\gamma_{i j}^{\kappa}$ are the macro-scale and micro-scale gradients, respectively of the average grain rotations and the fluctuations in grain rotations. For a material point, the macro-scale average displacement gradient and the average rotation gradient are taken as constants to ensure that the deformation mapping is single valued or uniquely invertible and the resultant kinematic fields satisfy certain compatibility conditions $[24,26]$. Consequently, their macro-scale gradients (i.e. the second macro-scale gradients of the average displacement and rotation fields) will vanish. Thus the second gradient of the grain displacement and rotation fields are found to be the macro-scale gradients of the micro-gradients $\gamma_{i j}^{\phi}$ and $\gamma_{i j}^{\kappa}$ as

$$
\begin{gathered}
\phi_{i, j k}=\psi_{i j, k}^{\phi}=\left(\bar{\phi}_{i, j}-\gamma_{i j}^{\phi}\right)_{, k}=-\gamma_{i j, k}^{\phi} \\
\kappa_{i, j k}=\psi_{i j, k}^{\kappa}=\left(\bar{\kappa}_{i, j}-\tilde{\kappa}_{i, j}\right)_{, k}=-\gamma_{i j, k}^{\kappa}
\end{gathered}
$$

Now combining Eqs. 1, 4, 6a and 7a, the relative displacement between neighboring grains $n$ and $p$ is obtained as

$$
\delta_{i}^{n p}=\phi_{i}^{p}-\phi_{i}^{n}=\phi_{i, j}^{n} l_{j}+\phi_{i, j k}^{n} J_{j k}=\bar{\phi}_{i, j} l_{j}-\gamma_{i j}^{\phi} l_{j}+\frac{1}{2} \psi_{i j, k}^{\phi} l_{j} l_{k}=\delta_{i}^{M}-\delta_{i}^{m}+\delta_{i}^{g}
$$

where

$\delta_{i}^{M}=\bar{\phi}_{i, j} l_{j} ; \quad \delta_{i}^{m}=\gamma_{i j}^{\phi} l_{j} ; \quad \delta_{i}^{g}=\frac{1}{2} \psi_{i j, k}^{\phi} l_{j} l_{k}=\psi_{i j, k}^{\phi} J_{j k}$

As seen from Eq. 9, the inter-granular relative displacements between two interacting (contacting) grains is decomposed into three terms: (1) $\delta_{i}^{M}$ due to the average displacement gradient, $\bar{\phi}_{i, j}$, (2) $\delta_{i}^{m}$ due to the gradients of the fluctuation in grain displacement, $\gamma_{i j}(x)$, and (3) $\delta_{i}^{g}$ due to a second gradient term $\psi_{i j, k}^{\phi}$.

Further, the relative rotation of two grains is also derived by combining Eqs. 1, 5, 6b and 7b, to obtain

$\theta_{i}^{n p}=\kappa_{i}^{p}-\kappa_{i}^{n}=\kappa_{i, j}^{n} l_{j}+\kappa_{i, j k}^{n} J_{j k}=\bar{\kappa}_{i, j} l_{j}-\gamma_{i j}^{\kappa} l_{j}+\frac{1}{2} \psi_{i j, k}^{\kappa} l_{j} l_{k}=\theta_{i}^{M}-\theta_{i}^{m}+\theta_{i}^{8}$

where

$\theta_{i}^{M}=\bar{\kappa}_{i, j} l_{j} ; \quad \theta_{i}^{m}=\gamma_{i j}^{\kappa} l_{j} ; \quad \theta_{i}^{g}=\frac{1}{2} \psi_{i j, k}^{\kappa} l_{j} l_{k}=\psi_{i j, k}^{\kappa} J_{j k}$

The inter-granular relative rotation has been also decomposed into three terms: (1) $\theta_{i}^{M}$ due to the gradients of the average rotations, (2) $\theta_{i}^{m}$ due to the gradient of the fluctuations in grain rotations, and (3) $\theta_{i}^{g}$ due to the second 
gradient of the rotation field of the grains. Since the second rank tensors $\gamma_{i j}^{\phi}\left(x_{k}\right)$ and $\gamma_{i j}^{k}\left(x_{k}\right)$ are independent of coordinates $x^{\prime}{ }_{k}$ (homogeneous within the material point), the fluctuation modeled in this way is taken as linear within the material point which leads to a micromorphic theory of degree 1 in the terminology introduced by Germain [28]. The decomposition of displacement gradient in Eq. 6 is supported by the experimentally measured kinematic fields of grain assemblies [46, 47, 51] that show strong non-affine motions in a volume element comprising large number of grains. The presence of grain displacement fluctuations have also been recognized in previous works related to continuum modeling of grain packing within the rubric of classical continuum mechanics [8,9] and becomes clear when we consider a discrete analysis of grain packing subjected to uniform classical kinematic boundary conditions. The second and higher gradient of deformations have also been shown to be necessary for continuum modeling of pantographic trusses due to their unique structural arrangements and stiffness of the truss elements $[52,53]$.

\section{Macro-scale stresses and inter-granular forces}

The deformation energy of a VE can now be expressed in two ways. One in terms of the macro-scale kinematic measures, and second as function of the grain-pair relative movements (the micro-scale kinematic measures). Thus the macro-scale deformation energy density of the granular continua can be defined as follows:

$W=W\left(\bar{\phi}_{i, j}, \gamma_{i j}^{\phi}, \psi_{i j, k}^{\phi}, \bar{\kappa}_{i, j}, \gamma_{i j}^{\kappa}, \psi_{i j, k}^{\kappa}\right)$

Conjugate to each one of the above kinematic fields, a macroscale stress measure are defined using the deformation energy density as:

$\begin{array}{lll}\tau_{i j}=\frac{\partial W}{\partial \bar{\phi}_{i, j}}=\frac{\partial W}{\partial \varepsilon_{i j}} ; & \sigma_{i j}=\frac{\partial W}{\partial \gamma_{i j}^{\phi}} ; & \mu_{i j k}=\frac{\partial W}{\partial \psi_{i j, k}^{\phi}} \\ M_{i j}=\frac{\partial W}{\partial \bar{\kappa}_{i, j}} ; & P_{i j}=\frac{\partial W}{\partial \gamma_{i j}^{\kappa}} ; & Q_{i j k}=\frac{\partial W}{\partial \psi_{i j, k}^{\kappa}}\end{array}$

where the stress measures conjugate to the displacement fields are shown with Greek letters while the moment stress measures conjugate to the spin fields are shown with Latin letters. $\tau_{i j}$ and $\sigma_{i j}$ are Cauchy stress and relative stress, respectively, while $\mu_{i j k}$ is the double stress tensor conjugate to the second gradient terms. Also the two second rank tensors, $M_{i j}$ and $P_{i j}$ are the average and relative moment stress tensors conjugate to the average and fluctuations in grain rotations respectively, while the third rank tensor, $Q_{i j k}$ is the double moment tensor conjugate to the second gradient of grain rotations.

The micro-scale deformation energy, $W^{\alpha}$, due to the relative displacements and relative rotations of grain-pairs, is defined as follows:for the $\alpha^{\text {th }}$ interacting grain-pair as a function of the micro-scale kinematic measures:
$W^{\alpha}\left(\delta_{i}^{M}, \delta_{i}^{m}, \delta_{i}^{g}, \theta_{i}^{M}, \theta_{i}^{m}, \theta_{i}^{g}\right)$,

where for simplicity of presentation the superscript $\alpha$ has been dropped from the micro-scale kinematic variables. The intergranular force and moment conjugates can now be introduced as derivatives of micro-scale deformation energy function, $W^{\alpha}$, with respect to each of the inter-particle kinematic measures as:

$$
\frac{\partial W^{\alpha}}{\partial \delta_{i}^{\alpha \xi}}=f_{i}^{\alpha \xi} ; \text { where } \xi: \mathrm{M}, \mathrm{m}, g \text { and } \frac{\partial W^{\alpha}}{\partial \theta_{i}^{\alpha \xi}}=m_{i}^{\alpha \xi} ; \text { where } \xi: \mathrm{M}, \mathrm{m}, g
$$

Note that these different forces and moments are work conjugates of their corresponding kinematic measures. At a given material point, that is on the VE of a granular material, if the kinematic field that corresponds to one of the displacement measures presented in Eq. 9 and 11 is imposed, the derivative of the resulting deformation energy with respect to that displacement/rotation measure will be equal to the conjugate force/moment measure. It is remarkable here that a notional total force equivalent to the sum of the above forces may be considered and for particular formulations of the micro-scale deformation energy function it may be possible to find such an equivalence. However, the definition of such a notional total force is moot due to the lack of a plausible identification of the total relative displacement with a single continuum kinematic counterpart, a key difficulty that makes the continuum modelling of discrete systems particularly challenging.

Further, the macro-scale deformation energy density, $W$, of the VE can be obtained as the volume average of the microscale deformation energy given by

$$
W=\frac{1}{V} \sum_{\alpha} W^{\alpha}\left(\delta_{i}^{M}, \delta_{i}^{m}, \delta_{i}^{g}, \theta_{i}^{M}, \theta_{i}^{m}, \theta_{i}^{g}\right)
$$

Combining Eqs. 12 through 13, applying chain rule of differentiation, and making use of the definition of microscopic kinematic measures in terms of their macroscopic counterparts given in Eqs. 9 and 11, the following expressions for the macro-scale stress measures in the terms of the intergranular force and moment conjugates can be derived:

$$
\left.\begin{array}{l}
\tau_{i j}=\frac{\partial W}{\partial \varepsilon_{i j}}=\frac{1}{V} \sum_{\alpha} \frac{\partial W^{\alpha}}{\partial \delta_{k}^{M}} \frac{\partial \delta_{k}^{M}}{\partial \varepsilon_{i j}} \\
\frac{\partial \delta_{k}^{M}}{\partial \varepsilon_{i j}}=\frac{\partial\left(\varepsilon_{k q} l_{q}^{\alpha}\right)}{\partial\left(\varepsilon_{i j}\right)}=\delta_{i k} \delta_{j q} l_{q}^{\alpha}=\delta_{i k} l_{j}^{\alpha}
\end{array}\right\} \Rightarrow \tau_{i j}=\frac{1}{V} \sum_{\alpha} \frac{\partial W^{\alpha}}{\partial \delta_{k}^{M}} \delta_{i k} l_{j}^{\alpha}=\frac{1}{V} \sum_{\alpha} f_{i}^{M} l_{j}^{\alpha}
$$

$$
\left.\begin{array}{l}
\sigma_{i j}=\frac{\partial W}{\partial \gamma_{i j}^{\phi}}=\frac{1}{V} \sum_{\alpha} \frac{\partial W^{\alpha}}{\partial \delta_{k}^{m}} \frac{\partial \delta_{k}^{m}}{\partial \gamma_{i j}^{u}} \\
\frac{\partial \delta_{k}^{m}}{\partial \gamma_{i j}^{\phi}}=\frac{\partial\left(\gamma_{k q}^{\phi} l_{q}^{\alpha}\right)}{\partial \gamma_{i j}^{\phi}}=\delta_{i k} \delta_{j q} l_{q}^{\alpha}=\delta_{i k} l_{j}^{\alpha}
\end{array}\right\} \Rightarrow \sigma_{i j}=\frac{1}{V} \sum_{\alpha} \frac{\partial W^{\alpha}}{\partial \delta_{k}^{m}} \delta_{i k} l_{j}^{\alpha}=\frac{1}{V} \sum_{\alpha} f_{i}^{m} l_{j}^{\alpha}
$$




$$
\begin{array}{r}
\mu_{i j k}=\frac{\partial W}{\partial \psi_{i j, k}^{\phi}}=\frac{1}{V} \sum_{\alpha} \frac{\partial W^{\alpha}}{\partial \delta_{l}^{g}} \frac{\partial \delta_{l}^{g}}{\partial \gamma_{i j}^{u}} \\
\left.\begin{array}{r}
\frac{\partial \delta_{l}^{g u}}{\partial \psi_{i j, k}^{\phi}}=\frac{\partial\left(\psi_{l, q r}^{\phi} J_{q r}^{\alpha}\right)}{\partial \psi_{i j, k}^{\phi}}=\delta_{i l} \delta_{j q} \delta_{k r} J_{q r}^{\alpha}=\delta_{i l} J_{j k}^{\alpha}
\end{array}\right\} \\
\Rightarrow \mu_{i j k}=\frac{1}{V} \sum_{\alpha} \frac{\partial W^{\alpha}}{\partial \delta_{k}^{g}} \delta_{i l} J_{j k}^{\alpha}=\frac{1}{V} \sum_{\alpha} f_{i}^{g} J_{j k}^{\alpha}
\end{array}
$$

$$
\left.\begin{array}{l}
M_{i j}=\frac{\partial W}{\partial \bar{\kappa}_{i, j}}=\frac{1}{V} \sum_{\alpha}\left(\frac{\partial W^{\alpha}}{\partial \theta_{k}^{M}} \frac{\partial \theta_{k}^{M}}{\partial \bar{\kappa}_{i, j}}\right) \\
\frac{\partial \theta_{k}^{M}}{\partial \bar{\kappa}_{i, j}}=\frac{\partial\left(\bar{\kappa}_{k, q} l_{q}\right)}{\partial \bar{\kappa}_{i, j}}=\delta_{i k} \delta_{j q} l_{q}^{\alpha}=\delta_{i k} l_{j}^{\alpha}
\end{array}\right\} \Rightarrow M_{i j}=\frac{1}{V} \sum_{\alpha}\left(\frac{\partial W^{\alpha}}{\partial \theta_{k}^{M}} \delta_{i k} l_{j}^{\alpha}\right)=\frac{1}{V} \sum_{\alpha} m_{i}^{M} l_{j}^{\alpha}
$$

$$
\left.\begin{array}{l}
P_{i j}=\frac{\partial W}{\partial \gamma_{i, j}^{\kappa}}=\frac{1}{V} \sum_{\alpha}\left(\frac{\partial W^{\alpha}}{\partial \theta_{k}^{m}} \frac{\partial \theta_{k}^{m}}{\partial \gamma_{i j}^{\kappa}}\right) \\
\frac{\partial \theta_{k}^{m}}{\partial \gamma_{i j}^{\omega}}=\frac{\partial\left(\gamma_{k q}^{\kappa} l_{q}\right)}{\partial \gamma_{i j}^{\kappa}}=\delta_{i k} \delta_{j q} l_{q}^{\alpha}=\delta_{i k} l_{j}^{\alpha}
\end{array}\right\} \Rightarrow P_{i j}=\frac{1}{V} \sum_{\alpha}\left(\frac{\partial W^{\alpha}}{\partial \theta_{k}^{m}} \delta_{i k} l_{j}^{\alpha}\right)=\frac{1}{V} \sum_{\alpha} m_{i}^{m} l_{j}^{\alpha}
$$

$$
\begin{array}{r}
Q_{i j k}=\frac{\partial W}{\partial \psi_{i j, k}^{\kappa}}=\frac{1}{V} \sum_{\alpha}\left(\frac{\partial W^{\alpha}}{\partial \theta_{l}^{g}} \frac{\partial \theta_{l}^{g}}{\partial \psi_{i j, k}^{\kappa}}\right) \\
\left.\begin{array}{r}
\frac{\partial \theta_{l}^{g}}{\partial \psi_{i j, k}^{\kappa}}=\frac{\partial\left(\psi_{l q, r}^{\kappa} J_{q r}^{\alpha}\right)}{\partial \psi_{i j, k}^{\kappa}}=\delta_{i l} \delta_{j q} \delta_{r k} J_{q r}^{\alpha}=\delta_{i l} J_{j k k}^{\alpha}
\end{array}\right\} \\
\Rightarrow Q_{i j k}=\frac{1}{V} \sum_{\alpha} \frac{\partial W^{\alpha}}{\partial \theta_{l}^{g}} \delta_{i l} J_{j k}^{\alpha}=\frac{1}{V} \sum_{\alpha} m_{i}^{g} J_{j k}^{\alpha}
\end{array}
$$

where we note again that the superscript $\alpha$ has been dropped from the micro-scale kinematic and force measure for readability. The macro-scale stress measures have been defined in terms of the inter-granular force measures and the local geometry represented by the branch vector, $l_{j}$, and geometry moment tensor, $J_{j k}$. We observe that the intergranular forces $f_{i}^{M}, f_{i}^{m}$, and $f_{i}^{g}$ are related to the Cauchy, the relative, and double moment stresses, respectively. Similarly, the inter-granular moments, $m_{i}^{M}, m_{i}^{m}$, and $m_{i}^{g}$ contribute to the average and relative moment stresses and double moment stress tensors, respectively. It is clear from Eq. 13 that the different force measures represent the change in grain-pair deformation energy with respect to the corresponding kinematic measures. Furthermore, grain-pair stiffness may be defined as the change in grain-pair force measures with respect to the micro-scale kinematic measures. The implication is clearly that separate grain-pair constitutive relations must be introduced corresponding to each of the force measures in terms of the micro-scale kinematic measures. The grain-pair constitutive relations cannot be estimated just from the behavior of two isolated grain since the neighborhoods and by extension the whole assembly affect the behavior of grain interactions. These grain-pair constitutive relations can then lead to a continuum constitutive law completing the micro-macro identification (see for example $[54,55]$ ). It is remarkable therefore, that the length-scale effects in granular material at the macro-scale, for which continuum models are relevant, arise from nonlocality and are not only functions of grain-size, but also of granular structure of the extended grain neighborhood and the inter-granular interaction mechanics. At the continuum scale, the length-scale effects are reflected in the elastic moduli and the dispersion relationships shown in the previous work presented by authors, in which grain spins were ignored [54, 55]. Finally, it is noteworthy that expression for some of the stress measures, particularly those in Eq. 15 through 17, in terms of grain pair forces have been reported in the literature $[48,56-59]$ as well as in context of atomic systems [41, 60] and are traceable to the virial theorem of Clausius [61]. The novelty of the expressions derived in this paper is the introduction of separate force measures conjugate to the grain-pair (micro-scale) counterparts of the continuum deformation measures.

\section{Summary and conclusion}

Accurate macro-scale description of the mechanical response of granular solids require the incorporation of the grain-scale mechanism in the continuum models. In this communication we have presented the kinematic variables necessary for determination of the grain-pair relative movement and their relationships to continuum counterparts. To this end two grain-scale kinematic measures, one for determining relative displacements and the other for relative rotations, have been defined. The introduced grain-scale kinematic measures represent the combined effect of the grain centroid displacement, spin and size, and do not follow the decomposition adopted in some previous attempts of micromacro identifications. In an extension of the previously presented the granular micromechanics approach, the grainscale motions are identified with continuum kinematic measures that include the usual macro-scale displacement /rotation gradients, micro-scale displacement /rotations gradients identified with displacement/rotation fluctuations within a material point, and macro-gradient of the micro-scale displacement/rotations gradients.

The deformation energy density of a material point is then expressed in terms of the kinematic measures at the twoscales and the inter-granular force measures as well as the continuum stress are defined as conjugates of the kinematic measures. Further, by identifying the micro- and macrodeformation energy densities, the relationships are derived between stress and inter-granular forces that include stretch/compression, tangential, bending and torsional actions. The approach outlined is reminiscent of developments in continuum modeling presented by Piola [22] which proceeds with the ansatz of specifying micro-motions in terms of the macro-motions and identifying the micro- and macro-works. This approach can form the basis for further derivation of the constitutive relations, variational principle, and balance equations for a non-classical micromorphic continuum model that incorporate grain-scale representation as described in [36]. 
Acknowledgements. This research is supported in part by the United States National Science Foundation grant CMMI1068528.

\section{References}

[1] C.S. Chang, A. Misra, Theoretical and Experimental-Study of Regular Packings of Granules, Journal of Engineering Mechanics-Asce 115(4) (1989) 704-720.

[2] C.S. Chang, A. Misra, Packing Structure and Mechanical-Properties of Granulates, Journal of Engineering Mechanics-Asce 116(5) (1990) 10771093.

[3] P.J. Digby, The Effective Elastic Moduli of Porous Granular Rocks, Journal Applied Mechanics 48 (1981) 803-808.

[4] K. Walton, The Effective Elastic Moduli of a Random Packing of Spheres, J. Mech.Phys.Solids 35 (1987) 213-226.

[5] H. Deresiewicz, Stress-Strain Relations for a Simple Model of a Granular Medium, Journal of Applied Mechanics 25 (1958) 402-406.

[6] J. Duffy, R.D. Mindlin, Stress-Strain Relations of a Granular Medium, Journal of Applied Mechanics 24(4) (1957) 585-593.

[7] J.T. Jenkins, Volume change in small strain axisymmetric defromations of a granular material, in: M. Satake, J.T. Jenkins (Eds.), Micromechanics of Granular Materials, Elsevier Science Publishers, Amsterdam, 1988, pp. $245-252$.

[8] A. Misra, C.S. Chang, Effective elastic moduli of heterogeneous granular solids, International Journal of Solids and Structures 30(18) (1993) 2547-2566.

[9] J. Jenkins, D. Johnson, L. La Ragione, H. Makse, Fluctuations and the effective moduli of an isotropic, random aggregate of identical, frictionless spheres, Journal of the Mechanics and Physics of Solids 53(1) (2005) $197-$ 225.

[10] N. Kruyt, L. Rothenburg, Kinematic and static assumptions for homogenization in micromechanics of granular materials, Mechanics of Materials 36(12) (2004) 1157-1173.

[11] F. Nicot, F. Darve, R. Group, A multi-scale approach to granular materials, Mechanics of materials 37(9) (2005) 980-1006.

[12] C. Chang, P.-Y. Hicher, An elasto-plastic model for granular materials with microstructural consideration, International journal of solids and structures 42(14) (2005) 4258-4277.

[13] K.L. Johnson, Contact mechanics, Cambridge University Press, Cambridge, U.K., 1985.

[14] A. Sutton, R. Balluffi, Interfaces in crystalline solids, Clarendon: Oxford (1995).

[15] A. Misra, P. Poorsolhjouy, Granular micromechanics model for damage and plasticity of cementitious materials based upon thermomechanics, Mathematics and Mechanics of Solids (2015) 1081286515576821.

[16] A. Misra, P. Poorsolhjouy, Granular micromechanics model of anisotropic elasticity derived from Gibbs potential, Acta Mechanica 227(5) (2016) 1393-1413.

[17] A. Misra, V. Singh, Thermomechanics-based nonlinear rate-dependent coupled damage-plasticity granular micromechanics model, Continuum Mech Therm 27(4-5) (2014) 787-817.

[18] C.L. Navier, Sur les lois de l'equilibre et du mouvement des corps solides elastiques, Memoire de l'Academie Royale de Sciences 7 (1827) 375-393.

[19] A.-L. Cauchy, Sur l'equilibre et le mouvement d'un systeme de points materiels sollicites par des forces d'attraction ou de repulsion mutuelle, Excercises de Mathematiques 3 (1826-1830) 188-212.

[20] F. dell'Isola, U. Andreaus, L. Placidi, At the origins and in the vanguard of peri-dynamics, non-local and higher gradient continuum mechanics. An underestimated and still topical contribution of Gabrio Piola, Mechanics and Mathematics of Solids (2013).

[21] N. Auffray, F. dell'Isola, V. Eremeyev, A. Madeo, G. Rosi, Analytical continuum mechanics a la Hamilton-Piola least action principle for second gradient continua and capillary fluids, Mathematics and Mechanics of Solids 20(4) (2013) 375-417.

[22] F. dell'Isola, G. Maier, U. Perego, U. Andreaus, R. Esposito, S. Forest, The complete works of Gabrio Piola: Volume I: Commented English
Translation-English and Italian Edition, Springer International Publishing2014.

[23] E. Cosserat, F. Cosserat, Theory of Deformable Bodies, SCIENTIFIC LIBRARY A. HERMANN AND SONS, Paris, 1909.

[24] R.D. Mindlin, Micro-Structure in Linear Elasticity, Archive for Rational Mechanics and Analysis 16(1) (1964) 51-78.

[25] R.A. Toupin, Theories of Elasticity with Couple-Stress, Archive for Rational Mechanics and Analysis 17(2) (1964) 85-112.

[26] A.C. Eringen, Microcontinuum field theories: foundations and solids, Springer New York1999.

[27] A.E. Green, R.S. Rivlin, Multipolar Continuum Mechanics, Archive for Rational Mechanics and Analysis 17(2) (1964) 113-147.

[28] P. Germain, Method of Virtual Power in Continuum Mechanics .2. Microstructure, Siam Journal on Applied Mathematics 25(3) (1973) 556575.

[29] H. AminPour, N. Rizzi, A one-dimensional continuum with microstructure for single-wall carbon nanotubes bifurcation analysis, Mathematics and Mechanics of Solids (2015) 1081286515577037.

[30] S. Gabriele, N. Rizzi, V. Varano, A one-dimensional nonlinear thin walled beam model derived from Koiter shell theory, Civil-Comp Proceedings (2014) 106.

[31] S. Gabriele, N. Rizzi, V. Varano, A 1D nonlinear TWB model accounting for in plane cross-section deformation, International Journal of Solids and Structures (2016).

[32] A. Madeo, P. Neff, I.-D. Ghiba, L. Placidi, G. Rosi, Wave propagation in relaxed micromorphic continua: modeling metamaterials with frequency band-gaps, Continuum Mech Therm (2013) 1-20.

[33] L. Placidi, U. Andreaus, A. Della Corte, T. Lekszycki, Gedanken experiments for the determination of two-dimensional linear second gradient elasticity coefficients, Zeitschrift für angewandte Mathematik und Physik 66(6) (2015) 3699-3725.

[34] J.-J. Alibert, A. Della Corte, Second-gradient continua as homogenized limit of pantographic microstructured plates: a rigorous proof, Zeitschrift für angewandte Mathematik und Physik 66(5) (2015) 2855-2870.

[35] S. Forest, Micromorphic approach for gradient elasticity, viscoplasticity, and damage, Journal of Engineering Mechanics 135(3) (2009) 117-131.

[36] A. Misra, P. Poorsolhjouy, Granular micromechanics based micromorphic model predicts frequency band gaps, Continuum Mech Therm 28(1-2) (2016) 215-234.

[37] Y. Yang, A. Misra, Micromechanics based second gradient continuum theory for shear band modeling in cohesive granular materials following damage elasticity, International Journal of Solids and Structures 49(18) (2012) 2500-2514.

[38] K.-C. Chen, J.-Y. Lan, Micromorphic modeling of granular dynamics, International Journal of Solids and Structures 46(6) (2009) 1554-1563.

[39] J. Goddard, From granular matter to generalized continuum, Mathematical Models of Granular Matter, Springer2008, pp. 1-22.

[40] H. Aminpour, N. Rizzi, On the Modelling of Carbon Nano Tubes as Generalized Continua, Generalized Continua as Models for Classical and Advanced Materials, Springer2016, pp. 15-35.

[41] Y. Chen, J.D. Lee, Connecting molecular dynamics to micromorphic theory.(I). Instantaneous and averaged mechanical variables, Physica A: Statistical Mechanics and its Applications 322 (2003) 359-376.

[42] F. dell'Isola, I. Giorgio, M. Pawlikowski, N. Rizzi, Large deformations of planar extensible beams and pantographic lattices: heuristic homogenization, experimental and numerical examples of equilibrium, Proc. R. Soc. A, The Royal Society, 2016, p. 20150790.

[43] D. Scerrato, I. Giorgio, N.L. Rizzi, Three-dimensional instabilities of pantographic sheets with parabolic lattices: numerical investigations, Zeitschrift für angewandte Mathematik und Physik 67(3) (2016) 1-19.

[44] D. Scerrato, I.A. Zhurba Eremeeva, T. Lekszycki, N.L. Rizzi, On the effect of shear stiffness on the plane deformation of linear second gradient pantographic sheets, ZAMM - Journal of Applied Mathematics and Mechanics/Zeitschrift für Angewandte Mathematik und Mechanik (2016).

[45] A. Misra, V. Singh, Nonlinear granular micromechanics model for multi-axial rate-dependent behavior, International Journal of Solids and Structures 51(13) (2014) 2272-2282.

[46] A. Misra, Particle kinematics in Sheared Rod Assemblies, Physics of Dry Granular Media, Springer1998, pp. 261-266.

[47] A. Misra, H. Jiang, Measured kinematic fields in the biaxial shear of granular materials, Computers and Geotechnics 20(3-4) (1997) 267-285. 
[48] A.S.J. Suiker, R. de Borst, C.S. Chang, Micro-mechanical modelling of granular material. Part 1: Derivation of a second-gradient micro-polar constitutive theory, Acta Mechanica 149(1-4) (2001) 161-180.

[49] M. Jiang, H.-S. Yu, D. Harris, Kinematic variables bridging discrete and continuum granular mechanics, Mechanics Research Communications 33(5) (2006) 651-666.

[50] L. Malvern, Introduction to Continuum Mechanics, Prentice Hall Inc., Engle Cliffs, NJ 1696 (1969) 301-320.

[51] V. Richefeu, G. Combe, G. Viggiani, An experimental assessment of displacement fluctuations in a 2D granular material subjected to shear, Geotechnique Letters 2 (2012) 113-118.

[52] J.J. Alibert, P. Seppecher, F. Dell'Isola, Truss modular beams with deformation energy depending on higher displacement gradients, Mathematics and Mechanics of Solids 8(1) (2003) 51-73.

[53] P. Seppecher, J.-J. Alibert, F. dell'Isola, Linear elastic trusses leading to continua with exotic mechanical interactions Journal of Physics: Conference Series 319(1) (2011) 012018.

[54] A. Misra, P. Poorsolhjouy, Identification of higher-order elastic constants for grain assemblies based upon granular micromechanics, Mathematics and Mechanics of Complex Systems 3(3) (2015) 285-308.
[55] A. Misra, P. Poorsolhjouy, Elastic Behavior of 2D Grain Packing Modeled as Micromorphic Media Based on Granular Micromechanics, Journal of Engineering Mechanics (2016) C4016005.

[56] J. Christoffersen, M. Mehrabadi, S. Nemat-Nasser, A micromechanical description of granular material behavior, Journal of Applied Mechanics 48(2) (1981) 339-344.

[57] J.H. Irving, J.G. Kirkwood, The Statistical Mechanical Theory of Transport Processes .4. The Equations of Hydrodynamics, Journal of Chemical Physics 18(6) (1950) 817-829.

[58] L. Rothenburg, A. Selvadurai, A micromechanical definition of the Cauchy stress tensor for particulate media, in: A. Selvadurai (Ed.) Mechanics of Structured Media, Elsevier, Amsterdam, 1981, pp. 496-486.

[59] J.-F. Ganghoffer, On the generalized virial theorem and Eshelby tensors, International Journal of Solids and Structures 47(9) (2010) 1209-1220.

[60] E.B. Tadmor, R.E. Miller, Modeling materials: continuum, atomistic and multiscale techniques, Cambridge University Press2011.

[61] R. Clausius, XVI. On a mechanical theorem applicable to heat, The London, Edinburgh, and Dublin Philosophical Magazine and Journal of Science 40(265) (1870) 122-127. 